This item was submitted to Loughborough's Research Repository by the author.

Items in Figshare are protected by copyright, with all rights reserved, unless otherwise indicated.

\title{
Spontaneous growth of bismuth nanowires on a sputter-deposited thin bismuth film
}

PLEASE CITE THE PUBLISHED VERSION

http://dx.doi.org/10.1016/j.surfcoat.2015.02.002

PUBLISHER

(C) Elsevier B.V.

VERSION

AM (Accepted Manuscript)

\section{PUBLISHER STATEMENT}

This work is made available according to the conditions of the Creative Commons Attribution-NonCommercialNoDerivatives 4.0 International (CC BY-NC-ND 4.0) licence. Full details of this licence are available at: https://creativecommons.org/licenses/by-nc-nd/4.0/

\section{LICENCE}

CC BY-NC-ND 4.0

\section{REPOSITORY RECORD}

Caruana, Andrew J., Michael D. Cropper, and Steven A. Stanley. 2019. "Spontaneous Growth of Bismuth Nanowires on a Sputter-deposited Thin Bismuth Film”. figshare. https://hdl.handle.net/2134/17658. 
Spontaneous growth of bismuth nanowires on a sputter-deposited thin bismuth film
A. J. Caruana, M. D. Cropper and S. A. Stanley

Department of Physics, Loughborough University

Loughborough,

LE11 3TU

United Kingdom

m.d.cropper@lboro.ac.uk

Thin films of bismuth of thickness $100 \mathrm{~nm}$ have been deposited onto $\mathrm{Si}(100)$ substrates held at $120^{\circ} \mathrm{C}$ using magnetron sputter deposition. The three-inch magnetrons were operated at DC powers between $30 \mathrm{~W}$ and $50 \mathrm{~W}$ to give deposition rates between 0.03 and $0.09 \mathrm{nms}^{-1}$. Examination of the surface of the thin films using scanning electron microscopy revealed nanowires of diameter $80-120 \mathrm{~nm}$ and length between 3 and $16 \mu \mathrm{m}$ emerging from the film. An investigation of the effect of deposition rate found little influence on the nanowire diameter, but indicated that some longer nanowires could be found on the film deposited at the lowest rate. A 20 $\mathrm{nm}$ film grown on glass at $110^{\circ} \mathrm{C}$ shows a nanowire with dimension around $50 \mathrm{~nm}$. Using a relatively higher rate for the first $25 \mathrm{~nm}$ followed by a lower rate to $100 \mathrm{~nm}$ produced some very straight and regular nanorods.

\section{Highlights:}

- $100 \mathrm{~nm}$ diameter bismuth nanowires made by sputter-deposition.

- Nanowires 3-16 micrometers long.

- Diameter not strongly deposition rate dependent in range $0.03-0.09 \mathrm{nms}^{-1}$.

- Commencing deposition with temporary higher rate gave straight nanorods.

Keywords: Bismuth; nanowires: sputter-deposition. 


\section{Introduction}

Bismuth is a group $V$ semi-metal that has been the focus of research spanning decades, motivated by several interesting properties that it possesses. Firstly, the carrier density of bismuth is much lower than the other metals, $10^{5}$ times smaller at liquid helium temperatures than in conventional metals, resulting in a relatively high resistivity [1]. Secondly, conduction electrons in bismuth have a very small effective mass. Thirdly they have a long carrier mean free path $(\sim 100 \mathrm{~nm}$ at $300 \mathrm{~K}$ and $\sim 400$ $\mu \mathrm{m}$ at $4 \mathrm{~K})[2]$ and finally the wavelength of electrons at the Fermi level is very long giving rise to more prominent quantum confinement effects in bismuth nanowires than in other materials. Bismuth has a small band overlap energy between its L-point conduction band and T-point valence band (38 meV at $77 \mathrm{~K}$ ) [3], anisotropic Fermi surface [4], low density of states [5] and high carrier mobility [6]. It is reported that the small overlap between the valence and conduction bands means that it may be converted from a semimetal to a semiconductor by reducing the dimensions of the material [7].

The crystal structure of bismuth is rhombohedral [8], but it is often described by reference to a hexagonal unit cell (or very occasionally by reference to a pseudocubic cell); here we will indicate planes and directions notated in the rhombohedral scheme by subscript $\mathrm{R}$ and in the hexagonal scheme by subscript $\mathrm{H}$. Bismuth has equal densities of holes and electrons, which cancels contributions to the Seebeck coefficient leading to bulk bismuth being a poor thermoelectric material [9]. Bismuth nanowires, however, are expected to have a good thermoelectric efficiency [4] due to the reduced dimensionality $[10,11,12]$. 
Given the long Fermi wavelength of bismuth, quantum confinement is expected in nanowires of quite large dimension $[13,14]$. There is a rapidly increasing interest in the production of bismuth nanowires as evidenced by the large number of publications in this field over the past few years. However, bismuth nanowires have been found to be difficult to fabricate as bismuth has a very low meting point $\left(271^{\circ} \mathrm{C}\right)$. Two broad groups of methods for their fabrication are template methods and on-film formation, each with its own inherent challenges and each producing nanowires with different crystallinities, regularity and diameter control.

Template methods may use either pressure injection [15] or electrochemical techniques to introduce bismuth into a porous alumina template or glass capillary. This method has good diameter control and the challenge is to produce nanowires with good regularity and crystallinity; developments over time have refined these methods. Pressure injection can result in single crystal nanowires grown along the length of the alumina template, but requires dissolving the template and manipulation of the solution in order to obtain single, separated nanowires [16]. Similar methods using electro-deposition have also been very successful [17, 18, 5] and good-quality nanowires have been manufactured by a template-free process [19].

Alternative methods for bismuth nanowire formation use physical vapour deposition (PVD). The attraction of PVD is that it can more readily produce structures that have high degrees of perfection in terms of crystallinity and regularity of the surface. One such PVD based method for nanowire production involves on film growth. In this method, nanowires are extruded from a bismuth film by post-deposition stress. One way to achieve this that has been reported involves the deposition of a bismuthchromium-nitride composite film, which extrudes bismuth nanowires due to stress 
retained within the film [20]. Another method involves the on-off process where a bismuth thin film is deposited onto a thermally oxidised silicon substrate [21]. After deposition the substrate is made to flex by cycling between high temperature and room temperature and the induced stress extrudes bismuth nanowires.

Recently there have been several reports of the spontaneous growth of bismuth nanowires and whiskers from sputter-deposited thin films without the requirement for post-deposition heating $[22,23,24,25,26]$. This PVD method requires the deposition of bismuth using magnetron sputtering at a low-rate onto a substrate that is maintained at an elevated temperature. If, for example, a $500 \mathrm{~nm}$ film is deposited then upon examination using scanning electron microscopy numerous whiskers or nanowires of length of several micro-meters are found to have grown from the films at a non-normal angle. The obvious attractions of this method of growing bismuth nanowires are that because the structures are produced by a natural crystallisation process they have a high degree of crystallinity, have smooth surfaces and can be manufactured relatively simply. The smoothness and crystallinity of these nanowires should make them suitable for investigations of fundamental physical properties.

\section{Examination of certain PVD grown nanowires using transmission electron} microscopy shows that they are crystalline with a $[110]_{H}$ growth direction $[22,25]$ but other reports suggest that $[012]_{\mathrm{H}}$ growth is also possible [26]. With low growth rates they emerge at angles around $54^{\circ}$ off-normal and appear to be promoted by the existence of $[110]_{R},[012]_{H}$ orientational texture in the film [23]. It has been suggested [25] that the growth direction precludes growth from the more dominant $[001]_{\mathrm{H}}$, $[111]_{R}$ texture that is particularly produced by evaporation deposition as the growth direction $[110]_{H}$ would then be within plane. For films grown on glass substrates it 
has also been pointed out that the thermal expansion coefficient of bismuth greatly exceeds that of glass so that cooling the sample from growth temperature to room temperature will not stress the film, making a stress-driven growth mechanism less likely [25]. The intriguing possibility that the whiskers grow in situ during film growth would be consistent with the observation that they grow on several different substrates [23].

Given the high degree of regularity apparent the method could be utilised to manufacture high-quality nanowires provided the control of diameter could be achieved. The growth mechanism of these whiskers is not known, but it has been proposed that they could grow around a spiral dislocation [22], or be the result of a catalyst free diffusion driven mechanism [23] such as asymmetric corner-crossing [26]. It has been reported that the film growth time [25] or growth rate [24] affects the diameter of the whiskers. The argument supporting the growth rate influence is that it has been shown that asymmetric corner-crossing produces bismuth nano-structures with an aspect ratio that is rate dependent [27]. These arguments suggest the intriguing possibility that by varying deposition conditions it may be possible to control the diameter of the nanowire produced. Nanowires emerging from a film deposited using RF sputtering at low rates have shown a variation in diameter with deposition rate [24]. In this work varying sputter power was used to grow films at rates between 0.044 and $0.341 \mathrm{nms}^{-1}$ for and deposition was for a period of 40 mins. The nanowires emerging these films had diameters between 20 and $400 \mathrm{~nm}$ respectively. Alternatively, in another report using slightly higher growth rate, a linear relationship was found between growth time and nanowire diameter in the range $100-600 \mathrm{~nm}$ at constant rate (though with a non-zero intercept) [25]. 
We have recently reported the growth of $500 \mathrm{~nm}$ diameter whiskers on $500 \mathrm{~nm}$ bismuth thin films [23] and indicated that nanowires may also be produced given a suitably thin film. Here we report on this phenomenon further, this time focussing on the production of nanowire diameter of order $100 \mathrm{~nm}$ or less. Given the importance of diameter control for the utility of this technique we have investigated the production of nanowires in the diameter range around $100 \mathrm{~nm}$ (and smaller) and the influence of growth parameters. We present an illustration of the utility of the use of the slow growth sputter-method for producing bismuth nanowires and nanorods.

\section{Experimental}

The bismuth nanowires grow naturally from crystallites in a bismuth thin film during deposition when the substrate temperature is suitably elevated. To enable formation of nanowires, it is important to grow the underlying films slowly. This was undertaken in a clean high vacuum physical vapour deposition system.

The bismuth thin films were deposited onto (100) oriented squares of silicon wafer of dimensions $20 \mathrm{~mm}$ by $20 \mathrm{~mm}$ (with the native oxide intact) or in one case onto glass. Prior to loading, the substrates were cleaned by degreasing and drying using acetone. The deposition was carried out using either pulsed DC or radio frequency (RF) planar magnetron sputtering in a thin films system manufactured by PVD Products Inc, Wilmington MA, USA. The vacuum chamber is made from stainless steel and is pumped by a rotary pump, turbo-molecular pump combination It can be subjected to a light "bake-out" using internal quartz heaters. The base pressure during the experiments was $2 \times 10^{-8}$ Torr which could be maintained by using a loadlock. 
The bismuth target was supplied by Testbourne Ltd and was of $99.99 \%$ purity. It was mounted into a 3" PVD Products Titan planar magnetron in the sputter-up configuration. In this system the axis of the magnetron is inclined at an angle of $35^{\circ}$ to the vertical and the target-substrate distance is $15 \mathrm{~cm}$. The power for the magnetron could be externally changed between DC and RF power. The DC power was supplied by an Advanced Energy MDX $1 K$ supply was used, combined with a Sparc-LE 20 unit in auto-run mode at $20 \mathrm{kHz}$ to supress arc formation [28]. Alternatively, RF power was supplied by a Dressler Cesar RF power generator. To ensure film uniformity the substrate was rotated at a rate of thirty times per minute. Before admitting the $99.9999 \%$ argon process gas the turbo-molecular pump was throttled and gas flow (and so sputtering pressure) was controlled by an MKS massflow controller.

To enable nanowire growth, the substrate temperature was held at $120^{\circ} \mathrm{C}$, except where otherwise stated. Heating was accomplished using a bank of quartz heaters behind the substrate and the temperature was measured using a thermocouple and controlled using a Eurotherm 2408 proportional-integral-derivative controller. The films deposited were $100 \mathrm{~nm}$ thick, except where otherwise stated, the deposition rate being measured by an Inficon XTC quartz crystal monitor. The rate monitor had previously been calibrated against measurements of film thickness using both X-ray reflectivity and scanning electron microscope of a fractured substrate imaged edgeon. Following deposition, films were examined using scanning electron microscopy (SEM), employing a Leo 1530FE high resolution field emission gun scanning electron microscope. 


\section{Results and Discussion}

The reports of the influence of growth rate [24] or that diameter is proportional to deposition time beyond a minimum of $171 \mathrm{~nm}$ when the film is grown at a rate between 1 and $4 \mathrm{nms}^{-1}$ [25] and that whiskers emerging from a $100 \mathrm{~nm}$ film have diameter of around $80 \mathrm{~nm}$ [23] pose the question: can film growth rate, growth time or film thickness be manipulated to give nanowires that are controllably in the range of $100 \mathrm{~nm}$ diameter or smaller? This latter, rather arbitrary figure was selected as it approximates to the Fermi wavelength in bismuth. To this end we have deposited and examined a series of $100 \mathrm{~nm}$ thick bismuth films deposited at different rates by varying the magnetron power with all other parameters held constant. In each case the films were grown to a specific thickness rather than for a specific time.

Figure 1 shows electron micrographs collected from bismuth films grown at different deposition rates. These were deposited onto sections of Si(100) wafer using DC magnetron sputtering. During deposition the substrate was held at $120^{\circ} \mathrm{C}$ and all the films are of $100 \mathrm{~nm}$ thickness. An important point that needs to be made is that compared with $500 \mathrm{~nm}$ films previously reported [23] the deposition of thinner films, gives typical nanowires of smaller diameter, but that the nanowires are sparser; the number density is not as high as it is on $500 \mathrm{~nm}$ films which have thicker whiskers.

Figure 1(a) shows a nanowire on a film grown using $50 \mathrm{~W}$ DC into a 3" target to give a rate of $0.09 \mathrm{nms}^{-1}$. This nanowire is $3 \mu \mathrm{m}$ long and just under $80 \mathrm{~nm}$ in diameter. Given a reliable method of harvesting, this would be of a reasonable size to use for investigation of quantum effects in the bismuth. The slight curve in the nanowire is common in these structures and is different from the behaviour seen in those of 500 $\mathrm{nm}$ diameter which are notably straight and rigid [23]. Figure 1(b) shows another 
image from the same film. This illustrates another feature that is frequently observed: the nanowire has changed direction in a well-defined manner. Such a clear-cut change of direction suggests a change in crystallographic growth direction, again providing evidence that a tip-growth model is more appropriate than a root-growth model. This would eliminate stress as a driver and suggest that surface diffusion is a more likely mechanism.

Figure 1(c) shows nanowires on a film grown using 40 W DC into a 3" target to give a deposition rate of $0.05 \mathrm{nms}^{-1}$. These nanowires are around $5 \mu \mathrm{m}$ and have similar diameters to that in Figure 1(a), but note that there are two different diameters visible in the micrograph. The range of diameters of nanowires found on this film is $80-140$ nm. Figure 1(d) shows an image of another nanowire on this film which also illustrates the occasional tendency for the growth direction to change. The angle of the change of direction here is about $53^{\circ}$ which is close to the angle between the two directions that have been exhibited as growth directions by bismuth nanowires [24, $25]-[110]_{H}$ and $[012]_{H}\left(\right.$ or $[10 \overline{1}]_{R}$ to $\left.[110]_{R}\right)$ - again emphasising that the origin of these structures most probably lies in growth rather than stress extrusion.

Figures $1(\mathrm{e})$ and (f) show nanowires on a film grown using 30 W DC into a 3" target to give a deposition rate of $0.03 \mathrm{nms}^{-1}$ : three times slower than in Figure 1(a). Again the nanowires have a similar diameter, but the length range is larger, extending from 7 to $16 \mu \mathrm{m}$. This suggests the possibility that depositing the underlying thin bismuth film at a low rate allows time for some very long nanowires to grow, which is consistent with the asymmetric corner crossing hypothesis [24, 27].

However, it appears that in this thickness and rate regime varying the deposition rate does not have a clear cut effect on nanowire diameter. It would appear that the 
nanowire diameter is primarily dependent on the total amount of bismuth deposited; that is the film thickness. It is of course likely that rate differences will also influence mean diameter and that other sputter deposition parameters could have an influence.

An illustration of the dependence of nanowire thickness on total bismuth deposition is illustrated in Figure 2. This shows a film deposited onto glass by RF sputtering at a rate of $0.04 \mathrm{nms}^{-1}$ to a total thickness of $20 \mathrm{~nm}$. Figure 2(a) shows a low magnification image of the film showing the paucity of nanowires when so little bismuth is deposited; this is a stark contrast to images collected from a $500 \mathrm{~nm}$ film [23], suggesting tha tnot all the whiskers begin growing at the same time during deposition; the longer a film grow for the more that may commence. This would complicate diameter control. Figure 1(b) shows a higher magnification of the same nanowire indicating a diameter of about $50 \mathrm{~nm}$. Thus the method may be used to grow quite narrow nanowires, albeit with a low yield.

The locus of growth of the nanowires does not appear to have any specific property, sometimes the wire grows out of the film and sometimes it is associated with one of the "hillocks". However, if the mechanism is growth rather than extrusion, it may be expected that the size of the film grains will have an effect on wire diameter. Depositing at higher rate is expected to give smaller film grains, so we investigated the effect of using two different powers during the same film growth: a high rate for the first $25 \mathrm{~nm}$ of deposition and then a lower rate. This was achieved by using $50 \mathrm{~W}$ DC magnetron power followed by $40 \mathrm{~W}$ or $30 \mathrm{~W}$. Figure 3(a-d) shows SEM images of some nanostructures found on these films. The nanowires measured on these films have length between 3 and $10 \mu \mathrm{m}$, but if anything have larger diameters than those deposited under single power - up to $140 \mathrm{~nm}$. Thus using a higher rate in this 
case has produced a larger diameter and shorter nanowire. One apparent feature of the nanowires on found on these films is that they are quite straight. Thus using a dual-power regime could be used to manufacture highly regular nanorods.

At the low rates used here, all the nanowires produced have very smooth sides. In the various reports of the production of these nanowires [22, 23, 26] there have been two distinct side wall structures: smooth and ridged. It has been suggested [26] that the ridged structures appear by growth around a dislocation and are $[110]_{\mathrm{H}}$ oriented, whereas the smooth surface nanowires appear by an asymmetric corner crossing method that promotes growth on the $[012]_{\mathrm{H}}$. It is surprising that two distinct growth mechanisms appear for the same material under similar conditions but either mechanism, it could be argued, appear because surface diffusion is the dominant factor in growth especially as the growth temperature is so close to the melting temperature. It is our experience that high-quality smooth-surfaced structures are favoured by very low deposition rates.

\section{Summary}

The spontaneous growth of bismuth whiskers on sputter-deposited thin films offers a route to manufacture nanowires of bismuth by controlling the growth conditions. We have deposited films of $100 \mathrm{~nm}$ thickness using deposition rates between 0.03 and $0.09 \mathrm{nms}^{-1}$ by varying the magnetron power. Images collected using SEM show that nanowires with diameters in the range $80-120 \mathrm{~nm}$ are produced, but with a lower areal density than on previously reported $500 \mathrm{~nm}$ films. These images suggest that the growth rate has much less influence on nanowire diameter than total film thickness, but that some much longer nanowires are to be found on thin films that are grown very slowly. A nanowire is shown emerging from a $20 \mathrm{~nm}$ film that is 
around $50 \mathrm{~nm}$ in diameter, showing that the method may be used to produce small

numbers of quite narrow nanowires. Using an initial higher deposition rate followed

by a slower one has produced nanowires that are very straight, but slightly thicker.

\section{Acknowledgements}

The authors would like to thank John Bates and Keith Yendall for assistance with the scanning electron microscopy and Simon Halse for assistance with some related experiments.

\section{References}

[1] T.W. Cornelius, M.E. Toimil-Molares, R. Neumann, S. Karim, J. Appl. Phys. 100 (2006) 114307.

[2] J. Heremans, C. Thrush, Y.-M. Lin, S. Cronin, Z. Zhang, M. Dresselhaus, J. Mansfield, Phys. Rev. B 61 (2000) 2921.

[3] J. Qi, D. Shi, J. Zhao, X. Jiang, J. Phys. Chem. C 112 (2008) 10745.

[4] Y.-T. Cheng, A.M. Weiner, C.A. Wong, M.P. Balogh, M.J. Lukitsch, Appl. Phys. Lett. 81 (2002) 3248.

[5] C.G. Jin, G.W. Jiang, W.F. Liu, W.L. Cai, L.Z. Yao, Z. Yao, X.G. Li, J. Mater. Chem. 13 (2003) 1743.

[6] Y. Wang, K.S. Kim, Nanotechnology 19 (2008) 265303.

[7] Y.-M. Lin, X. Sun, M.S. Dresselhaus1,2, Phys. Rev. B 62 (2000) 4610.

[8] R. W. G. Wyckoff, Crystal Structures Volume 1, 2nd Ed., Interscience, New York, 1963.

[9] J. Reppert, R. Rao, M. Skove, J. He, M. Craps, T. Tritt, A.M. Rao, Chem. Phys. Lett. 442 (2007) 334.

[10] E.J. Menke, M.A. Brown, Q. Li, J.C. Hemminger, R.M. Penner, Langmuir 22 (2006) 10564.

[11] L. Hicks, M. Dresselhaus, Phys. Rev. B 47 (1993) 12727.

[12] D. Gitsu, L. Konopko, A. Nikolaeva, T.E. Huber, Appl. Phys. Lett. 86 (2005) 102105.

[13] T.E. Huber, A. Nikolaeva, D. Gitsu, L. Konopko, M.J. Graf, Phys. E Low-Dimensional Syst. Nanostructures 37 (2007) 194.

[14] P. Chiu, I. Shih, Nanotechnology 15 (2004) 1489.

[15] M. Gurvitch, J. Low Temp. Phys. 38 (1980) 777.

[16] J. Heremans, C. Thrush, Y.-M. Lin, S. Cronin, Z. Zhang, M. Dresselhaus, J. Mansfield, Phys. Rev. B 61 (2000) 2921.

[17] K. Liu, C. Chien, P. Searson, Phys. Rev. B 58 (1998) R14681.

[18] L. Li, Y. Zhang, G. Li, L. Zhang, Chem. Phys. Lett. 378 (2003) 244.

[19] Y. Xu, Z. Ren, W. Ren, G. Cao, K. Deng, Y. Zhong, Nanotechnology 19 (2008) 115602.

[20] Y.-T. Cheng, A.M. Weiner, C.A. Wong, M.P. Balogh, M.J. Lukitsch, Appl. Phys. Lett. 81 (2002) 3248.

[21] W. Y. Shim, K. I. Lee, J. Y. Chang, S. H. Han, W. Y. Jeung, Electron. Mater. Lett. 2 (2006) 33.

[22] S. Cao, C. Guo, Y. Wang, J. Miao, Z. Zhang, Q. Liu, Solid State Commun. 149 (2009) 87.

[23] S.A. Stanley, C. Stuttle, A.J. Caruana, M.D. Cropper, A.S.O. Walton, J. Phys. D. Appl. Phys. 45 (2012) 435304.

[24] Y. Tian, C. Fei Guo, S. Guo, Y. Wang, J. Miao, Q. Wang, Q. Liu, AIP Adv. 2 (2012) 012112.

[25] B.-K. Wu, H.-Y. Lee, M.-Y. Chern, Appl. Phys. Express 6 (2013) 035504.

[26] Y. Tian, L. Jiang, X. Zhang, Y. Deng, S. Deng, Mater. Res. Express 1 (2014) 035034. 
[27] D.N. McCarthy, S.A. Brown, J. Phys. Conf. Ser. 100 (2008) 072007.

[28] J. Li, M. K. Narasimhan, V. Pavate, D. Loo, S. Rosenblum, L. Trubell, R. Scholl, S. Seamons, C. Hagerty, S. Ramaswami, Integrated arc suppression unit for defect reduction in PVD applications, in: D. N. Patel and M. Graef (Eds.), Proc. SPIE 3214, Multilevel Interconnect Technology, International Society for Optics and Photonics, Washington, 1997, pp. 33-41. 


\section{Figure Captions}

Figure 1: SEM images of $100 \mathrm{~nm}$ bismuth films deposited onto $\mathrm{Si}(100)$ held at $120^{\circ} \mathrm{C}$ using DC magnetron sputtering. (a-b) Using $50 \mathrm{~W}$ to give a growth rate of $0.09 \mathrm{nms}^{-1}$, (c-d) using $40 \mathrm{~W}$ to give $0.05 \mathrm{nms}^{-1}$, (e-f) using $30 \mathrm{~W}$ to give $0.03 \mathrm{nms}^{-1}$.

Figure 2: SEM images of $25 \mathrm{~nm}$ bismuth films deposited onto glass held at $110^{\circ} \mathrm{C}$ using RF magnetron sputtering.

Figure 3: SEM images of $100 \mathrm{~nm}$ bismuth films deposited onto $\mathrm{Si}(100)$ held at $120^{\circ} \mathrm{C}$ using DC magnetron sputtering at two sequential powers: (a-b) $50 \mathrm{~W}$ for $25 \mathrm{~nm}$ then $30 \mathrm{~W}$; (c-d) $50 \mathrm{~W}$ for $25 \mathrm{~nm}$ then $40 \mathrm{~W}$ 


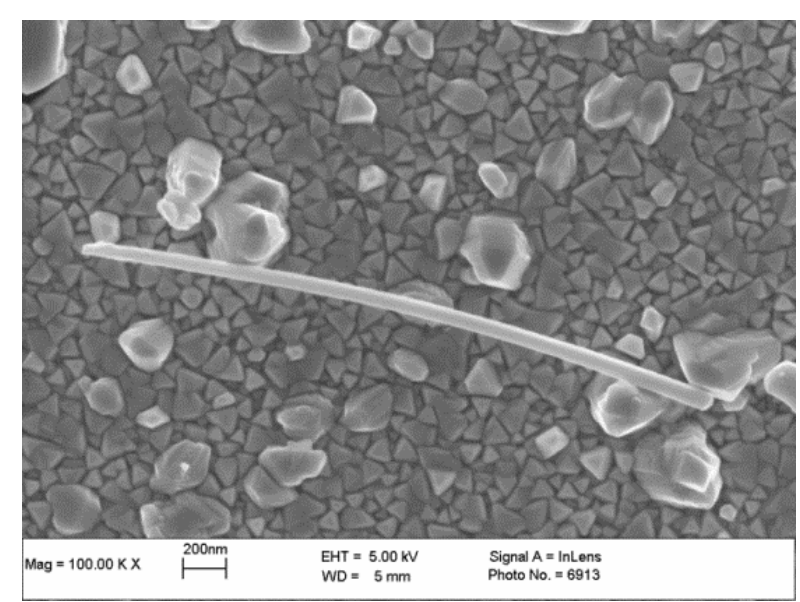

(a)

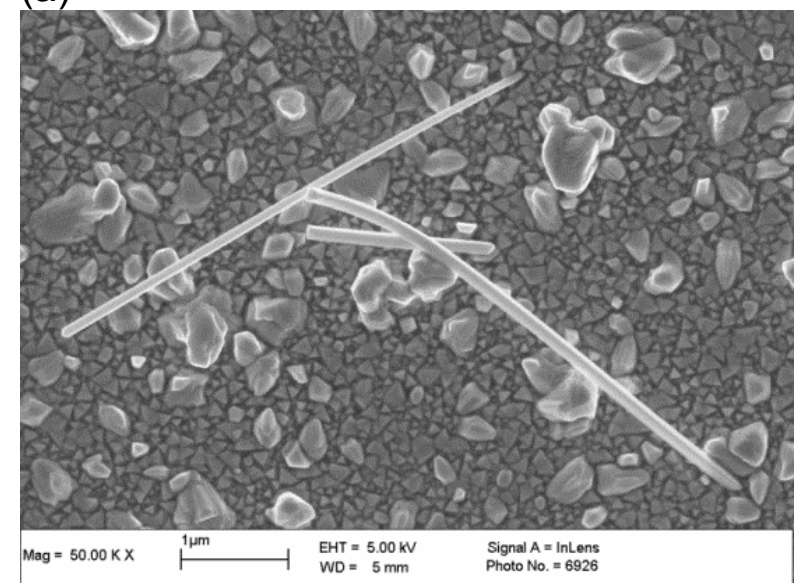

(c)

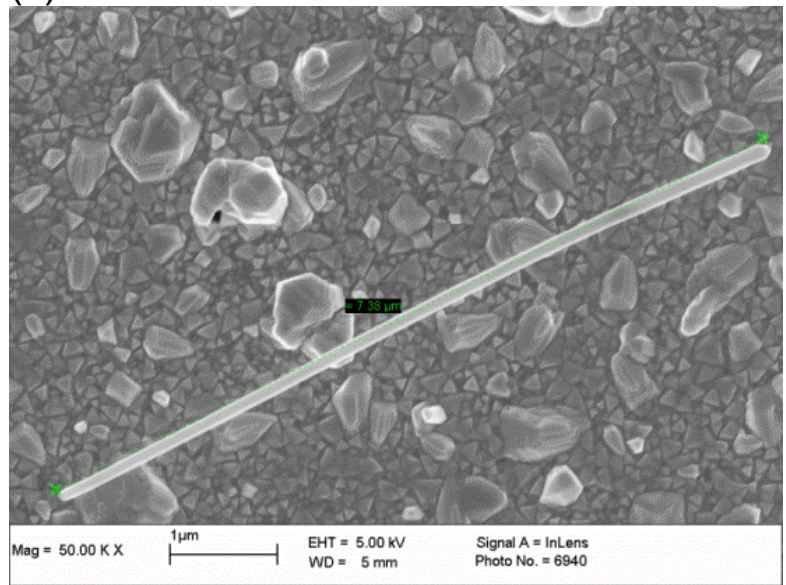

(e)

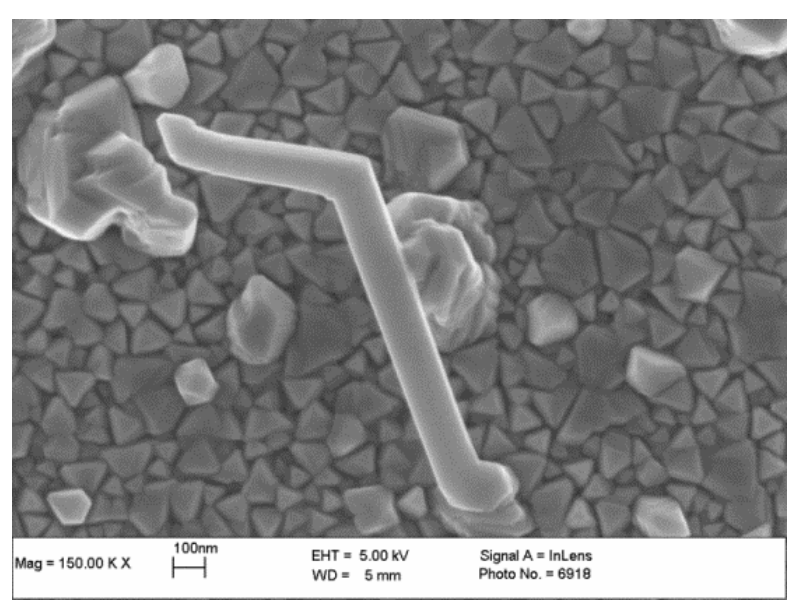

(b)

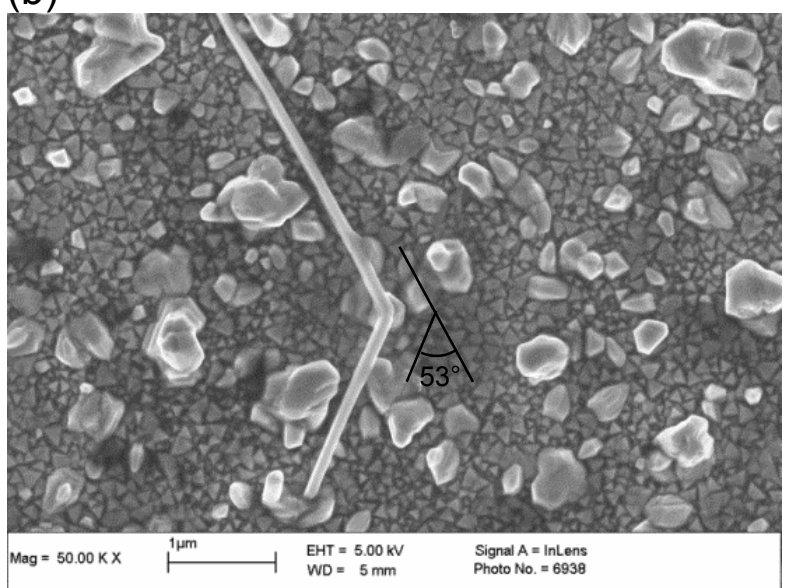

(d)

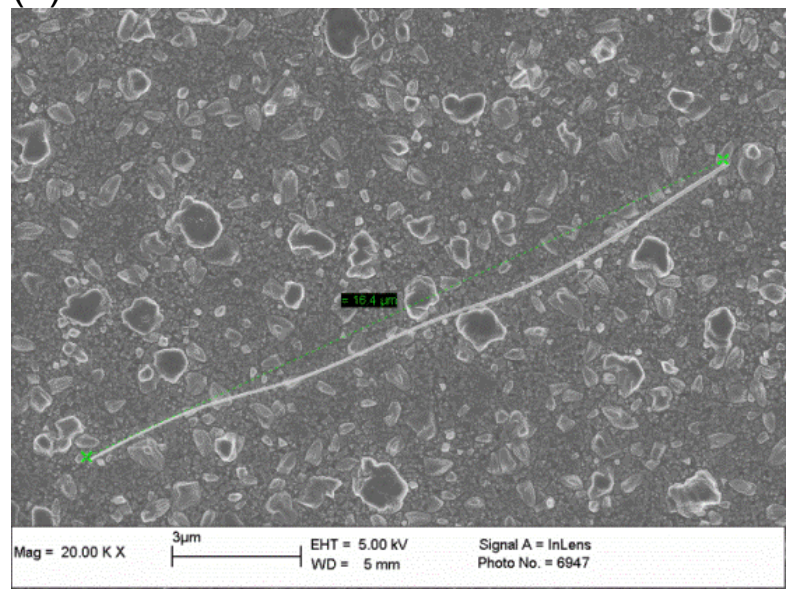

(f)

Figure 1 


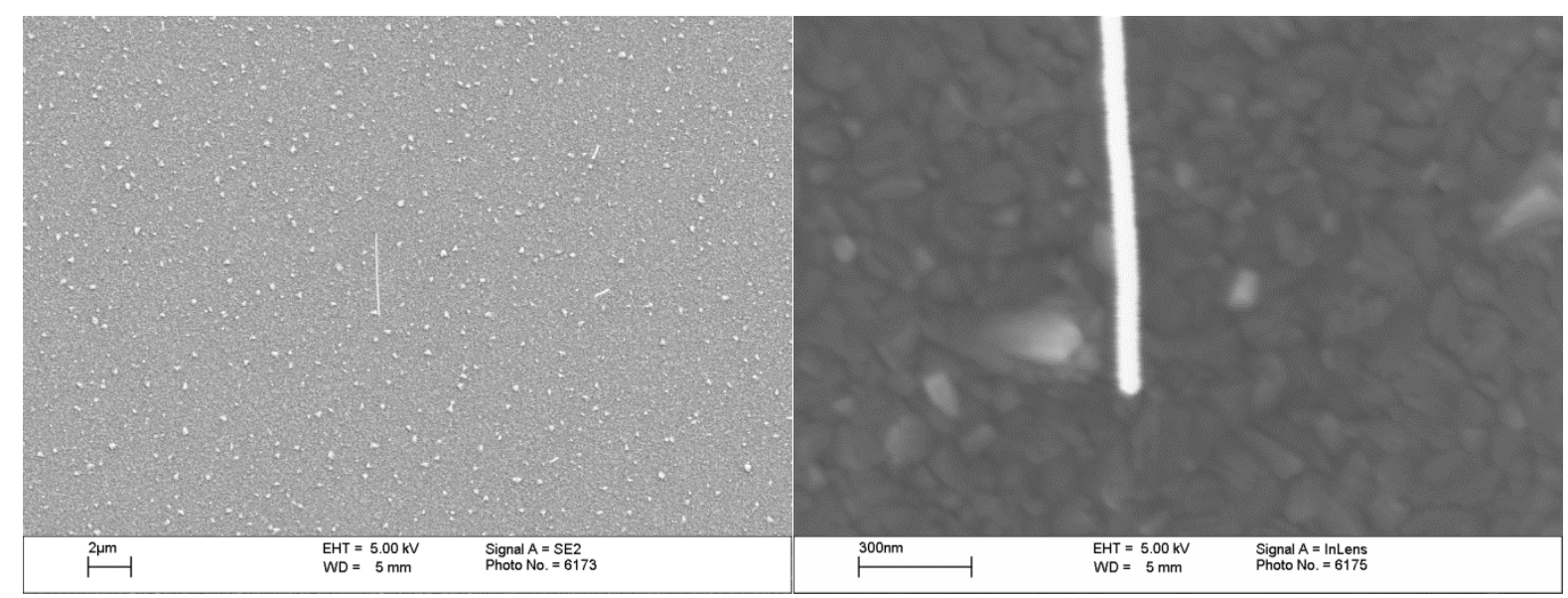

(a)

(b)

Figure 2 

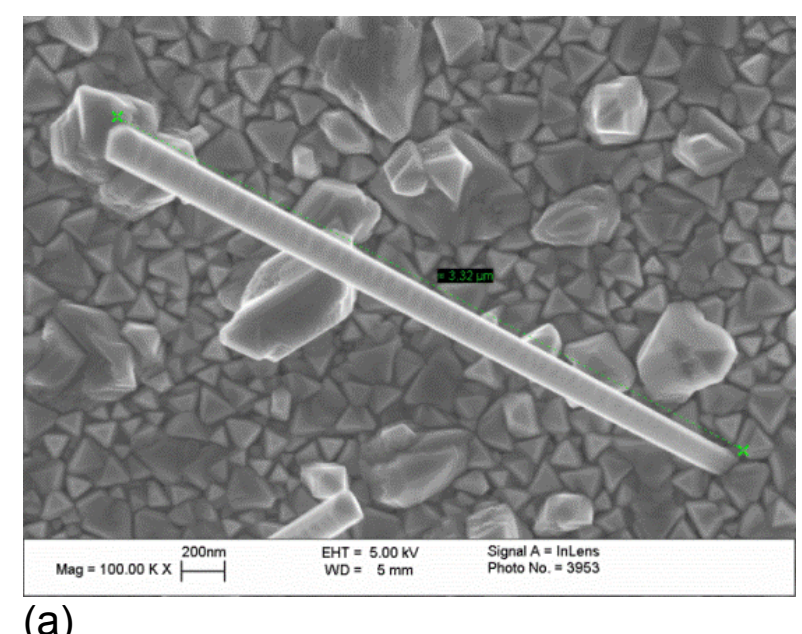

(a)

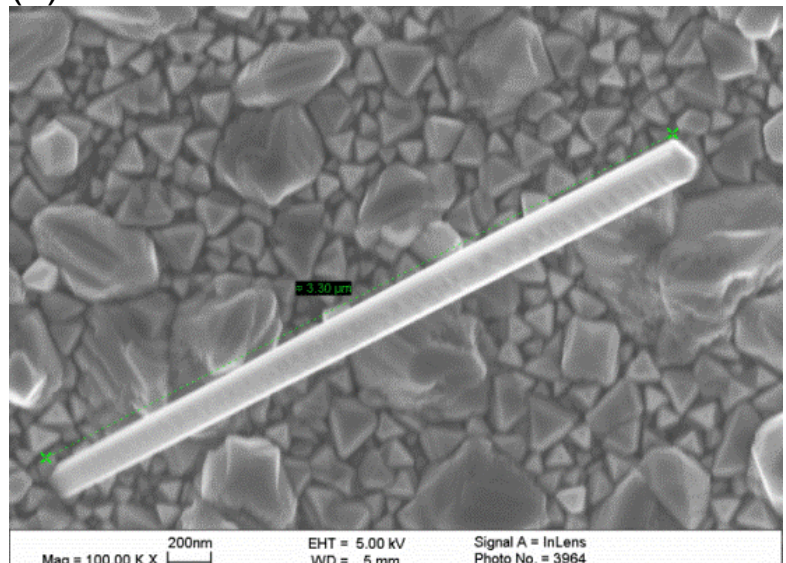

$\operatorname{mag}=100.00 \mathrm{~K} \times \stackrel{200 \mathrm{~mm}}{\longmapsto}$

(c)

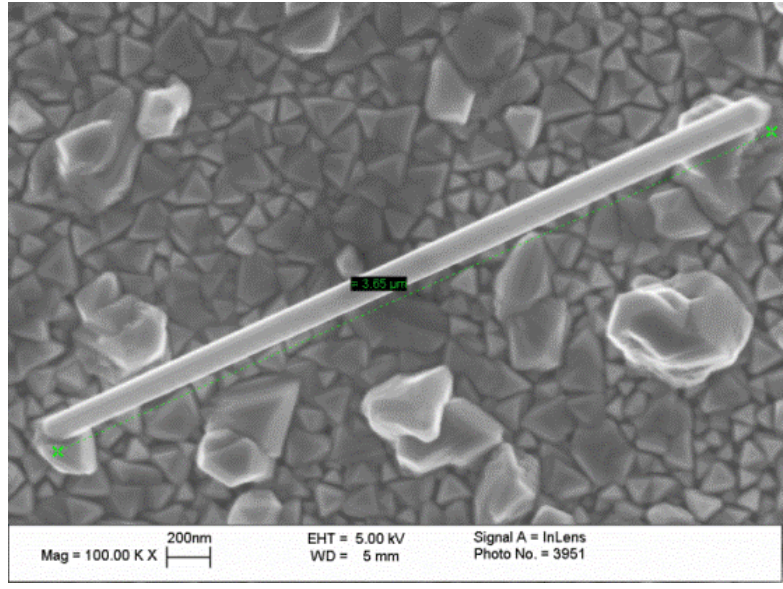

(b)

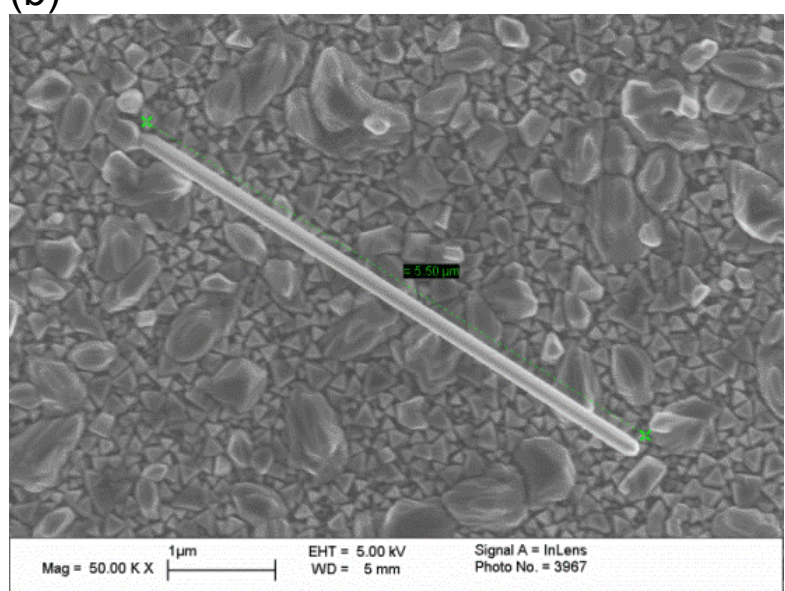

(d)

Figure 3 